\title{
PARTIAL DIFFERENTIAL EQUATIONS WITH DELAYED RANDOM PERTURBATIONS: EXISTENCE, UNIQUENESS AND STABILITY OF SOLUTIONS
}

\author{
Tomás Caraballo and José Real \\ Dpto. Análisis Matemático. Universidad de Sevilla \\ Apartado de Correos 1160. (41080)-Sevilla (Spain)
}

\begin{abstract}
We consider a stochastic non-linear Partial Differential Equation with delay which may be regarded as a perturbed equation. First, we prove the existence and the uniqueness of solutions. Next, we obtain some stability results in order to prove the following: if the unperturbed equation is exponentially stable and the stochastic perturbation is small enough then, the perturbed equations remains exponentially stable. We impose standard assumptions on the differential operators and we use strong and mild solutions.
\end{abstract}

\section{INTRODUCTION AND STATEMENT OF THE PROBLEM}

The aim of this paper is to analyze the behaviour of the solutions of Partial Differential Equations under the action of random perturbations. Nominally: if the solutions of a PDE are exponentially stable, under what kind of perturbations the solutions of the perturbed equation have the same kind of stability?

This problem has been investigated by many authors. We mention Haussmann [7], Curtain and Pritchard [5], Ichikawa [8], Chow [4], Mao [9,10], Zabczyk [14], Caraballo [2], among others. In this paper we will investigate the exponential stability of a stochastic PDE in relation to delay stochastic perturbations.

Let $V$ and $H$ be two separable Hilbert spaces with norms $\|\cdot\|$ and $|\cdot|$ respectively. Assume that $V$ is densely and continuously imbedded in $H$. We identify $H$ with its dual space $H^{\prime}$ :

$$
V \hookrightarrow H \hookrightarrow V^{\prime}
$$

and we denote by $(\cdot, \cdot)$ the inner product in $H$ and by $\langle\cdot, \cdot\rangle$ the duality between $V^{\prime}$ and $V\left(\left\langle x \in V^{\prime}, y \in V\right\rangle\right)$. 
Let $w_{t}$ be a Wiener process defined over the complete probability space $\left(\Omega, \mathcal{F}, P,\left\{\mathcal{F}_{t}\right\}_{t \geq 0}\right)$ and taking values into the separable Hilbert space $K$, where $\mathcal{F}_{t}$ is the $\sigma$-field generated by $\left\{w_{s}, 0 \leq s \leq t\right\}$. Let $W$ be the incremental covariance operator of $w_{t}$, which is a trace class operator on $K$ defined by

$$
W x=\sum_{i=1}^{\infty} \lambda_{i}\left(x, e_{i}\right) e_{i}, \quad x \in K
$$

and

$$
\operatorname{tr}(W)=\sum_{i=1}^{\infty} \lambda_{i}
$$

where $\left\{e_{i}\right\}$ is a complete orthonormal set in $K,\left\{\lambda_{i}\right\}$ is a sequence of nonnegative numbers such that $\sum \lambda_{i}<\infty$ and $\operatorname{tr}$ denotes the trace of an operator (see $\left.[8,11]\right)$. We also denote by $|\cdot|$ the norm in $\mathcal{L}(K, H)$.

We assume that $A: V \longrightarrow V^{\prime}$ is a linear continuous operator (i.e. $A \in \mathcal{L}\left(V, V^{\prime}\right)$ ) satisfying a coercivity condition:

(coerc)

$$
\exists \nu \in \mathbf{R}, \varepsilon>0: \quad-2\langle A x, x\rangle+\nu|x|^{2} \geq \varepsilon\|x\|^{2} \quad \forall x \in V .
$$

Let $B$ be an operator from $H$ into $\mathcal{L}(K, H)$ satisfying $B(0)=0$, and we assume there exists a positive constant $b$ such that

$$
|B(x)-B(y)| \leq b|x-y|, \quad \forall x, y \in H .
$$

Let $\rho:[0,+\infty) \longrightarrow \mathbf{R}$ be a continuously differentiable function (of delay) such that

$$
\exists h>0: \quad-h \leq \rho(t) \leq t, \quad \rho^{\prime}(t) \geq 1, \quad \forall t \geq 0,
$$

which obviously implies that there exists a positive constant $k$ with

$$
\rho^{-1}(t) \leq t+k, \quad \forall t \geq-h,
$$

and let $\psi$ be a function such that

$$
\psi \in I^{2}(-h, 0 ; H) \cap L^{2}(\Omega ; C(-h, 0 ; H)),
$$

where, for $T \geq 0$ fixed, by $L^{2}(\Omega ; C(-h, T ; H))$ we denote $L^{2}(\Omega, \mathcal{F}, d P ; C([-h, T] ; H))$, and by $I^{2}(-h, T ; H)$ the closed subspace of $L^{2}(\Omega \times[-h, T], \mathcal{F} \otimes \mathcal{B}[-h, T], d P \otimes d t ; H)$ of process $\mathcal{F}_{t}$-adapted for all $t \in[-h, T]$, where $\mathcal{F}_{t}=\mathcal{F}_{0}$ if $t \leq 0$. In Section 2 we prove: 
THEOREM 1.-Assume (coerc), (1.1) - (1.4). Then, there exists a unique process $x(t)$ which is solution of the problem:

$$
\left\{\begin{array}{lll}
x(t) \in I^{2}(-h, T ; H) \cap L^{2}(\Omega ; C(-h, T ; H)), & & \forall T \geq 0, \\
x(t)=\psi(0)+\int_{0}^{t} A x(s) d s+\int_{0}^{t} B(x(s)-x(\rho(s))) d w_{s}, & P-\text { a.s. }, \quad \forall t \geq 0, \\
x(t)=\psi(t), & \forall t \in[-h, 0] .
\end{array}\right.
$$

In the sequel, we shall write $(P C)$ in the following form:

$$
(P C)^{\prime} \quad \begin{cases}x(t) \in I^{2}(-h, T ; H) \cap L^{2}(\Omega ; C(-h, T ; H)), & \forall T>0, \\ d x(t)=A x(t) d t+B(x(t)-x(\rho(t))) d w_{t}, & \forall t \in[0, T], \\ x(t)=\psi(t), & \forall t \in[-h, 0] .\end{cases}
$$

Such a process is called the strong solution of $(P C)$. Note that if condition (coerc) is satisfied, $A$ generates a strongly continuous semigroup $S(t)$ in $H$ (see Dautray and Lions $[6])$, and the strong solution of $(P C)$ is also the mild solution. In other words, the strong solution $x(t)$ satisfies

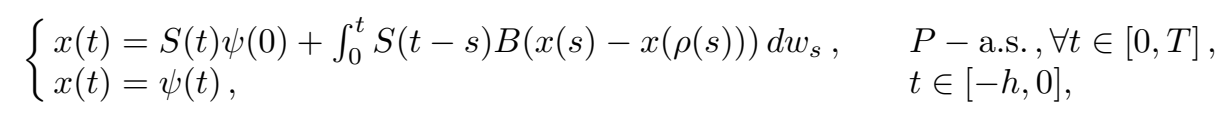

(see Chojnowska-Michalik [3] and Ichikawa [8]).

The problem $(P C)$ might be regarded as a stochastic perturbed system of the following linear differential system

$$
\frac{d x(t)}{d t}=A x(t) \quad \text { on } t \geq 0
$$

We assume the equation (1.6) is exponentially stable, that is,

$$
\exists M, \gamma>0 \text { such that }|S(t)|^{2} \leq M e^{-\gamma t}, \quad \forall t \geq 0,
$$

and we will prove (see Section 3) that the zero solution of the problem $(P C)$ also is exponentially stable provided the perturbation small enough. In fact, we will prove the following:

THEOREM 2.-Assume (coerc),(1.1) - (1.4), (A1) and (A2), where

$$
4 M b^{2} \operatorname{tr}(W)<\gamma .
$$

Then, there exist positive constants $\lambda, C$ such that

$$
E|x(t)|^{2} \leq C\|\psi\|_{1}^{2} e^{-\lambda t}, \quad \forall t \geq 0,
$$


where $\|\psi\|_{1}^{2}=\max \left\{E|\psi(0)|^{2}, \int_{-h}^{0} E|\psi(s)|^{2} d s\right\}$.

Haussmann [7] have studied this problem when the perturbation is $B x(t) d w_{t}$ for $B$ linear and bounded. If $|B|$ is small (i.e. verifies a condition similar to ( $A 2)$ ), Haussmann proves that the perturbed system remains exponentially stable. Ichikawa [8] does the same when the perturbation is $f(x(t)) d t+B(x(t)) d w_{t}$, where $f: H \rightarrow H$ and $B: H \rightarrow \mathcal{L}(K, H)$ are Lipschitz continuous.

However, the disturbances appearing in the problem are often affected by delay terms since the past has influence on the evolution of the process we are studying. For example, in Caraballo [2] is considered $B(x(\rho(t))) d w_{t}$ with linear continuous $B$ and is obtained exponential stability provided $|B|$ small enough, and the results are more general than Haussmann's since it holds for all delay function satisfying (1.2). Mao $[9,10]$ considers the term $B(x(t)-x(t-h)) d w_{t}$ for $h>0$, and obtains exponential stability when $A$ is a linear bounded operator in $H$ (i.e. (1.6) is a differential system but not a partial differential one) and $h$ is small enough. When $A$ is unbounded (i.e. (1.6) is a partial differential equation) and $B$ is linear, Mao can not ensure that for small $h$ the perturbed equation remains stable. However, Theorem 2 proves that the trivial solution of $(P C)$ is exponentially stable even though $B$ is not linear, $\rho$ satisfies (1.2) and $b$ is small .

In Section 2, we state the existence and uniqueness of solution for $(P C)$. We prove the main result of this paper in Section 3. We give some remarks and comments in Section 4. Finally, an example is given in order to illustrate our results.

\section{EXISTENCE AND UNIQUENESS OF SOLUTION}

Now, we are going to prove Theorem 1.

Uniqueness: Let $x, y \in I^{p}(-h, T ; V) \cap L^{2}(\Omega ; C(-h, T ; H))$ be two solutions of $(P C)$, then

$$
\begin{aligned}
x(t)-y(t)= & \int_{0}^{t} A(x(s)-y(s)) d s \\
& +\int_{0}^{t}(B(x(s)-x(\rho(s)))-B(y(s)-y(\rho(s)))) d w_{s} .
\end{aligned}
$$


Applying Itô's formula (see Pardoux [12]), using (coerc) and (1.1), we obtain

$$
E|x(t)-y(t)|^{2} \leq\left(\nu+2 b^{2}\right) \int_{0}^{t} E|x(s)-y(s)|^{2} d s+2 b^{2} \int_{0}^{t} E|x(\rho(s))-y(\rho(s))|^{2} d s
$$

Now, if we set $z(t)=x(t)-y(t)$, it follows from $(2.2)$

$$
E|z(t)|^{2} \leq\left(\nu+4 b^{2}\right) \int_{0}^{t} \sup _{r \in[0, s]} E|z(r)|^{2} d s,
$$

since $z(\rho(s))=0$ for $\rho(s) \leq 0$ and $\rho(s) \leq s$ for $s \geq 0$. Consequently, (2.3) yields

$$
\sup _{r \in[0, t]} E|z(r)|^{2} \leq\left(|\nu|+4 b^{2}\right) \int_{0}^{t} \sup _{r \in[0, s]} E|z(r)|^{2} d s .
$$

Finally, Gronwall's lemma implies

$$
\sup _{r \in[0, t]} E|z(r)|^{2}=0, \quad \forall t \in[0, T] .
$$

Uniqueness follows from (2.5).

Existence: We are going to prove existence of solutions in two different ways. In the first, we only need to use that $\rho$ is measurable and satisfies

$$
\exists h>0, \quad-h \leq \rho(t) \leq t \quad \forall t \geq 0,
$$

instead of $\rho$ is continuously differentiable and verifies (1.2). This fact permits to extend this technique to more general delay equation (see also Remark 1).

In the second one, we use condition (1.2) to give an easy proof which only is valid in this case.

FIRST PROOF: We consider the following equations

$$
\begin{aligned}
x^{1}(t) & =\psi(0)+\int_{0}^{t} A x^{1}(s) d s, \quad t \geq 0, \quad x^{1}(t)=\psi(t), \quad t \in[-h, 0] \\
x^{n+1}(t) & =\psi(0)+\int_{0}^{t} A x^{n+1}(s) d s-\frac{\nu}{2} \int_{0}^{t} x^{n+1}(s) d s \\
& +\int_{0}^{t} B\left(x^{n}(s)-x^{n}(\rho(s)) d w_{s}+\frac{\nu}{2} \int_{0}^{t} x^{n}(s) d s, \quad t \geq 0, n \geq 1\right.
\end{aligned}
$$

$$
x^{n+1}(t)=\psi(t), \quad t \in[-h, 0], \forall n \geq 1
$$


It follows from Pardoux [12, Theorem 1.1] that there exists a unique process $x^{1} \in I^{2}(-h, T ; V) \cap L^{2}(\Omega ; C(-h, T ; H))$ which is solution of equations (2.6). Since (1.1) implies that $B\left(x^{n}()-.x^{n}(\rho()).\right) \in I^{2}(0, T ; H)$ provided $x^{n} \in I^{2}(0, T ; V)$ and $\rho$ measurable, we then can get (again from Pardoux [12, Theorem 1.1]) a sequence

$$
x^{n} \in I^{2}(-h, T ; V) \cap L^{2}(\Omega ; C(-h, T ; H))
$$

such that $x^{1}$ is solution of (2.6) and $x^{n+1}$ is solution of (2.7)-(2.8) for $n \geq 1$.

Now, we will prove that $\left\{x^{n}\right\}_{n \geq 1}$ is a Cauchy sequence in $L^{2}(\Omega ; C(-h, T ; H))$ and in $I^{2}(-h, T ; V)$, and so it will converge to a process $x \in I^{2}(-h, T ; V) \cap L^{2}(\Omega ; C(-h, T ; H))$. Finally, since the linearity of $A$ and the Lipschitz continuity of $B$ permit us to take limits in $(2.6)-(2.8)$, we then obtain that $x$ is the solution of $(P C)$.

On the one hand, for $n>1$, it follows

$$
\begin{aligned}
x^{n+1}(t)-x^{n}(t) & =\int_{0}^{t} A\left(x^{n+1}-x^{n}\right) d s-\frac{\nu}{2} \int_{0}^{t}\left(x^{n+1}-x^{n}\right) d s \\
& +\frac{\nu}{2} \int_{0}^{t}\left(x^{n}-x^{n-1}\right) d s+\int_{0}^{t}\left[B\left(x^{n}-x_{\rho}^{n}\right)-B\left(x^{n-1}-x_{\rho}^{n-1}\right)\right] d w_{s},
\end{aligned}
$$

where, for short, we denote: $x^{n}:=x^{n}(s), x_{\rho}^{n}:=x^{n}(\rho(s))$.

Itô's formula for the process $x^{n+1}(t)-x^{n}(t)$ yields

$$
\begin{aligned}
& \left|x^{n+1}(t)-x^{n}(t)\right|^{2} \\
& =2 \int_{0}^{t}\left\langle A\left(x^{n+1}-x^{n}\right), x^{n+1}-x^{n}\right\rangle d s-\nu \int_{0}^{t}\left|x^{n+1}-x^{n}\right|^{2} d s \\
& +2 \int_{0}^{t}\left(x^{n+1}-x^{n},\left(B\left(x^{n}-x_{\rho}^{n}\right)-B\left(x^{n-1}-x_{\rho}^{n-1}\right)\right) d w_{s} i g h t\right) \\
& +\nu \int_{0}^{t}\left(x^{n+1}-x^{n}, x^{n}-x^{n-1}\right) d s \\
& +\int_{0}^{t} \operatorname{tr}\left[\left(B\left(x^{n}-x_{\rho}^{n}\right)-B\left(x^{n-1}-x_{\rho}^{n-1}\right)\right) W\left(B\left(x^{n}-x_{\rho}^{n}\right)-B\left(x^{n-1}-x_{\rho}^{n-1}\right)\right)^{*}\right] d s .
\end{aligned}
$$

The coercivity condition (coerc) implies 


$$
\begin{aligned}
& \left|x^{n+1}(t)-x^{n}(t)\right|^{2} \\
& \leq|\nu| \int_{0}^{t}\left|x^{n+1}-x^{n}\right|\left|x^{n}-x^{n-1}\right| d s \\
& +2\left|\int_{0}^{t}\left(x^{n+1}-x^{n},\left(B\left(x^{n}-x_{\rho}^{n}\right)-B\left(x^{n-1}-x_{\rho}^{n-1}\right)\right) d w_{s}\right)\right| \\
& +\int_{0}^{t}\left|\operatorname{tr}\left[\left(B\left(x^{n}-x_{\rho}^{n}\right)-B\left(x^{n-1}-x_{\rho}^{n-1}\right)\right) W\left(B\left(x^{n}-x_{\rho}^{n}\right)-B\left(x^{n-1}-x_{\rho}^{n-1}\right)\right)^{*}\right]\right| d s .
\end{aligned}
$$

Consequently, from (2.11) it holds

$$
\begin{aligned}
& E\left[\sup _{0 \leq \theta \leq t}\left|x^{n+1}(\theta)-x^{n}(\theta)\right|^{2}\right] \\
& \quad \leq|\nu| E \int_{0}^{t}\left|x^{n+1}-x^{n}\right|\left|x^{n}-x^{n-1}\right| d s \\
& \quad+\operatorname{tr}(W) E \int_{0}^{t}\left|B\left(x^{n}-x_{\rho}^{n}\right)-B\left(x^{n-1}-x_{\rho}^{n-1}\right)\right|^{2} d s \\
& \quad+2 E\left[\sup _{0 \leq \theta \leq t}\left|\int_{0}^{t}\left(x^{n+1}-x^{n},\left(B\left(x^{n}-x_{\rho}^{n}\right)-B\left(x^{n-1}-x_{\rho}^{n-1}\right)\right) d w_{s}\right)\right|\right] .
\end{aligned}
$$

Now, we evaluate the terms on the right-hand side of (2.12):

$$
\begin{aligned}
& |\nu| E \int_{0}^{t}\left|x^{n+1}-x^{n}\right|\left|x^{n}-x^{n-1}\right| d s \\
& \leq \frac{1}{4 T} E \int_{0}^{t}\left|x^{n+1}-x^{n}\right|^{2} d s+\nu^{2} T E \int_{0}^{t}\left|x^{n}-x^{n-1}\right|^{2} d s \\
& \leq \frac{1}{4} E\left[\sup _{0 \leq \theta \leq t}\left|x^{n+1}(\theta)-x^{n}(\theta)\right|^{2}\right] \\
& +\nu^{2} T \int_{0}^{t} E\left[\sup _{0 \leq \theta \leq s}\left|x^{n}(\theta)-x^{n-1}(\theta)\right|^{2}\right] d s . \\
& \operatorname{tr}(W) E \int_{0}^{t}\left|B\left(x^{n}-x_{\rho}^{n}\right)-B\left(x^{n-1}-x_{\rho}^{n-1}\right)\right|^{2} d s \\
& \leq \operatorname{tr}(W) b^{2} \int_{0}^{t} E\left|x^{n}-x_{\rho}^{n}-x^{n-1}+x_{\rho}^{n-1}\right|^{2} d s \\
& \leq 2 \operatorname{tr}(W) b^{2} \int_{0}^{t}\left(E\left|x^{n}-x^{n-1}\right|^{2}+E\left|x_{\rho}^{n}-x_{\rho}^{n-1}\right|^{2}\right) d s \\
& \leq 4 \operatorname{tr}(W) b^{2} \int_{0}^{t} E\left[\sup _{0 \leq \theta \leq s}\left|x^{n}(\theta)-x^{n-1}(\theta)\right|^{2}\right] d s .
\end{aligned}
$$


Burkholder-Davis-Gundy's inequality yields

$$
\begin{aligned}
& 2 E\left[\sup _{0 \leq \theta \leq t}\left|\int_{0}^{t}\left(x^{n+1}-x^{n},\left(B\left(x^{n}-x_{\rho}^{n}\right)-B\left(x^{n-1}-x_{\rho}^{n-1}\right)\right), d w_{s}\right)\right|\right] \\
& \leq 6 \operatorname{tr}(W)^{1 / 2} E\left[\int_{0}^{t}\left|B\left(x^{n}-x_{\rho}^{n}\right)-B\left(x^{n-1}-x_{\rho}^{n-1}\right)\right|^{2}\left|x^{n+1}-x^{n}\right|^{2} d s\right]^{1 / 2} \\
& \leq 6 \operatorname{tr}(W)^{1 / 2} E\left[\left(\sup _{0 \leq \theta \leq t}\left|x^{n+1}(\theta)-x^{n}(\theta)\right|^{2}\right)\right. \\
& \left.\quad \times \int_{0}^{t}\left|B\left(x^{n}-x_{\rho}^{n}\right)-B\left(x^{n-1}-x_{\rho}^{n-1}\right)\right|^{2} d s\right]^{1 / 2} \\
& \leq \frac{1}{4} E\left[\sup _{0 \leq \theta \leq t}\left|x^{n+1}(\theta)-x^{n}(\theta)\right|^{2}\right] \\
& +36 \operatorname{tr}(W) b^{2} E \int_{0}^{t}\left|x^{n}-x_{\rho}^{n}-x^{n-1}+x_{\rho}^{n-1}\right|^{2} d s \\
& \leq \\
& \quad \frac{1}{4} E\left[\sup _{0 \leq \theta \leq t}\left|x^{n+1}(\theta)-x^{n}(\theta)\right|^{2}\right] \\
& \quad+144 \operatorname{tr}(W) b^{2} \int_{0}^{t} E\left[\sup _{0 \leq \theta \leq s}\left|x^{n}(\theta)-x^{n-1}(\theta)\right|^{2}\right] d s .
\end{aligned}
$$

If we set

$$
\varphi^{n}(t)=E\left[\sup _{0 \leq \theta \leq t}\left|x^{n+1}(\theta)-x^{n}(\theta)\right|^{2}\right]
$$

then, there exists $c_{1}>0$ such that $(2.13)-(2.15)$ yield

$$
\varphi^{n}(t) \leq c_{1} \int_{0}^{t} \varphi^{n-1}(s) d s, \quad 0 \leq t \leq T .
$$

By iteration, we get from (2.17)

$$
\varphi^{n}(t) \leq \frac{c_{1}^{n-1} T^{n-1}}{(n-1) !} \varphi^{1}(T), \quad \forall n>1, \quad \forall t \in[0, T] .
$$

Thus $\left\{x^{n}\right\}$ is a Cauchy sequence in $L^{2}(\Omega ; C(-h, T ; H))$.

On the other hand, using (coerc) and (1.1), it follows from (2.10)

(2.19) $E\left|x^{n+1}(t)-x^{n}(t)\right|^{2}+\varepsilon E \int_{0}^{t}\left\|x^{n+1}(s)-x^{n}(s)\right\|^{2} d s$

$$
\begin{aligned}
& \leq|\nu| E \int_{0}^{t}\left|x^{n+1}(s)-x^{n}(s)\right|\left|x^{n}(s)-x^{n-1}(s)\right| d s \\
& +E \int_{0}^{t}\left|B\left(x^{n}-x_{\rho}^{n}\right)-B\left(x^{n-1}-x_{\rho}^{n-1}\right)\right|^{2} d s
\end{aligned}
$$




$$
\begin{aligned}
& \leq \frac{\nu^{2}}{2} E \int_{0}^{t} \sup _{0 \leq \theta \leq s}\left|x^{n}(\theta)-x^{n-1}(\theta)\right|^{2} d s+\frac{1}{2} E \int_{0}^{t} \sup _{0 \leq \theta \leq s}\left|x^{n+1}(\theta)-x^{n}(\theta)\right|^{2} d s \\
& +4 b^{2} E \int_{0}^{t} \sup _{0 \leq \theta \leq s}\left|x^{n}(\theta)-x^{n-1}(\theta)\right|^{2} d s \\
& \leq\left(\frac{\nu^{2}}{2}+4 b^{2}\right) T \varphi^{n-1}(t)+\frac{1}{2} T \varphi^{n}(t),
\end{aligned}
$$

and then, there exist positive constants $c_{2}, c_{3}$ such that

$$
E \int_{0}^{t}\left\|x^{n+1}(s)-x^{n}(s)\right\|^{2} d s \leq\left[c_{2} \frac{c_{1}^{n-1} T^{n}}{(n-1) !}+c_{3} \frac{c_{1}^{n-1} T^{n}}{(n-2) !}\right] \varphi^{1}(T) .
$$

Therefore, $\left\{x^{n}\right\}$ is a Cauchy sequence in $I^{2}(-h, T ; V)$.

In conclusion, it follows that

$$
\begin{aligned}
x^{n} & \rightarrow x \text { in } I^{2}(-h, T ; V) \cap L^{2}(\Omega ; C(-h, T ; H)) \\
A x^{n} & \rightarrow A x \text { in } I^{2}\left(0, T ; V^{\prime}\right) \\
x^{n}(\rho(.)) & \rightarrow x(\rho(.)) \text { in } L^{2}(\Omega ; C(0, T ; H)) \\
B\left(x^{n}(.)-x^{n}(\rho(.))\right) & \rightarrow B(x(.)-x(\rho(.))) \text { in } I^{2}(0, T ; H)
\end{aligned}
$$

We can thus take limits in (2.7)-(2.8) and it then follows that $x$ is the solution of $(P C)$.

SECOND PROOF: We observe that (1.2) implies that the function $\tau(t)=$ $\rho(t)-t$ is nondecreasing and non-positive, since $\tau^{\prime}(t)=\rho^{\prime}(t)-1 \geq 0$ and $\rho(t) \leq t$. So there exist only three cases:

$$
\text { Case (i): } \lim _{t \rightarrow+\infty}(\rho(t)-t)=-\delta<0 .
$$

Then, we consider equation $(P C)$

$$
x(t)=\psi(0)+\int_{0}^{t} A x(s) d s+\int_{0}^{t} B(x(s)-x(\rho(s))) d w_{s} \quad, \text { on } 0 \leq t \leq \delta .
$$

Since $\tau$ is nondecreasing, it follows that $-\delta=\sup _{t \in[0,+\infty)} \tau(t)$ and $\rho(t)-t \leq-\delta$ for all $t \geq 0$. So, $\rho(s) \leq s-\delta \leq 0$ for $0 \leq s \leq \delta$ and the equation $(P C)$ is now

$$
x(t)=\psi(0)+\int_{0}^{t} A x(s) d s+\int_{0}^{t} B(x(s)-\psi(\rho(s))) d w_{s} \quad \text { on } 0 \leq t \leq \delta,
$$

which is a non-delay equation and it is well known (see, for instance, Pardoux [11,12]) that there exists a unique solution on $0 \leq t \leq \delta$. By induction, equation $(P C)$ can be solved on $[k \delta,(k+1) \delta]$ for all $k \geq 0$ and therefore on $[0,+\infty)$. 
Case (ii): $\lim _{t \rightarrow+\infty}(\rho(t)-t)=0$, but $\rho(t)-t<0$ for any $t \geq 0$

In this case, we can choose an increasing sequence $\left\{t_{k}\right\}_{k \geq 0}$ such that $t_{0}=0, t_{k} \uparrow+\infty$ and $\rho\left(t_{k+1}\right) \leq t_{k}$. So equation $(P C)$ can be solved on $\left[t_{k}, t_{k+1}\right]$ for all $k \geq 0$ and therefore on $[0,+\infty)$.

Case (iii): There exists $T>0$ such that $\rho(t)<t$ for $t<T$ but $\rho(t)=t$ for all $t \geq T$.

Now we can solve $(P C)$ on $[0, T)$ in the same way as Case (ii). It is not difficult to show that $x(t) \longrightarrow x(T) \in L^{2}\left(\Omega, \mathcal{F}_{T}, d P ; H\right)$ when $t \rightarrow T$. Now, on $[T,+\infty),(P C)$ becomes

$$
x(t)=x(T)+\int_{T}^{t} A x(s) d s
$$

which has a unique solution obviously.

Remark 1. The technique used in the first proof for the existence is similar to that in Real [13], where is considered the linear case for a rather general equation with several delay functions and non-continuous martingales.

\section{PROOF OF THEOREM 2}

We shall split the proof in two steps. In the first one, we deduce that there exist positive constants $\lambda, k_{1}$ such that

$$
I:=\int_{0}^{\infty} e^{\lambda t} E\left|x_{t}\right|^{2} d t \leq k_{1}\|\psi\|_{1}^{2} .
$$

In the second, using (3.1) and Itô's formula we obtain (1.7).

First step: First, we observe that $I$ denotes the following

$$
I=\lim _{T \rightarrow \infty} \int_{0}^{T} e^{\lambda t} E|x(t)|^{2} d t=\lim _{T \rightarrow \infty} I(T) .
$$

Next, we note that this limit always exists (finite or not) since $e^{\lambda t} E|x(t)|^{2} \geq 0, \forall t \geq 0$.

Now, let $x(t)$ be the solution of $(P C)$. Then, we can write

$$
\left\{\begin{array}{l}
x(t)=S(t) \psi(0)+\int_{0}^{t} S(t-s) B(x(s)-x(\rho(s))) d w_{s}, P-a . s . \quad \forall t \geq 0 \\
x(t)=\psi(t), \quad t \in[-h, 0] .
\end{array}\right.
$$


¿From $(A 2)$ we can take $\lambda \in \mathbf{R}$ such that $0<\lambda<\gamma, 2 M b^{2} \operatorname{tr}(W)\left(1+e^{\lambda k}\right)<\gamma-\lambda$. Hence,

$$
\begin{aligned}
|x(t)|^{2}= & |S(t) \psi(0)|^{2}+\left|\int_{0}^{t} S(t-s) B(x(s)-x(\rho(s))) d w_{s}\right|^{2} \\
& +2\left(S(t) \psi(0), \int_{0}^{t} S(t-s) B(x(s)-x(\rho(s))) d w_{s}\right), P-a . s ., \quad \forall t \geq 0 .
\end{aligned}
$$

Taking expectations in (3.2), multiplying by $e^{\lambda t}$ and taking into account that $S(t) \psi(0)$ is $\mathcal{F}_{0}$-measurable, it then follows that, for each $T>0$,

$$
\begin{aligned}
\int_{0}^{T} e^{\lambda t} E|x(t)|^{2} d t & =\int_{0}^{T} e^{\lambda t} E|S(t) \psi(0)|^{2} d t \\
& +\operatorname{tr}(W) \int_{0}^{T} e^{\lambda t} \int_{0}^{t} E|S(t-s) B(x(s)-x(\rho(s)))|^{2} d s d t .
\end{aligned}
$$

Evaluating the terms on the right-hand side of (3.3), we obtain:

$$
\int_{0}^{T} e^{\lambda t} E|S(t) \psi(0)|^{2} d t \leq \frac{M}{\gamma-\lambda}\|\psi\|_{1}^{2}
$$

by $(A 1)$. Also, Fubini's Theorem, (1.1) and the change of variables $u=\rho(s)$ yield

$$
\begin{aligned}
& \int_{0}^{T} e^{\lambda t} \int_{0}^{t} E|S(t-s) B(x(s)-x(\rho(s)))|^{2} d s d t \\
& =\int_{0}^{T} \int_{s}^{T} e^{\lambda t} E|S(t-s) B(x(s)-x(\rho(s)))|^{2} d t d s \\
& \leq \int_{0}^{T} e^{\lambda s} \int_{0}^{T} e^{\lambda t} E|S(t) B(x(s)-x(\rho(s)))|^{2} d t d s \\
& \leq M b^{2} \int_{0}^{T} e^{(\lambda-\gamma) t} d t \int_{0}^{T} e^{\lambda s} E|x(s)-x(\rho(s))|^{2} d s \\
& \leq \frac{2 M b^{2}}{\gamma-\lambda}\left[\int_{0}^{T} e^{\lambda s} E|x(s)|^{2} d s+\int_{0}^{T} e^{\lambda s} E|x(\rho(s))|^{2} d s\right] \\
& \leq \frac{2 M b^{2} e^{\lambda k} h}{\gamma-\lambda}\|\psi\|_{1}^{2}+\frac{2 M b^{2}\left(1+e^{\lambda k}\right)}{\gamma-\lambda} \int_{0}^{T} e^{\lambda s} E|x(s)|^{2} d s .
\end{aligned}
$$

¿From (3.3)-(3.5),

$$
I(T)=\int_{0}^{T} e^{\lambda t} E|x(t)|^{2} d t \leq c_{1}\|\psi\|_{1}^{2}+c_{2} I(T)
$$

where $c_{2}<1$. Since $c_{1}$ and $c_{2}$ do not depend on $\mathrm{T}$, we have

$$
I(T) \leq \frac{c_{1}}{1-c_{2}}\|\psi\|_{1}^{2}, \quad \forall T>0,
$$


and taking limit for $T \rightarrow \infty$, we obtain that $I$ is finite and (3.1) holds.

Second step: Applying Itô's formula for the process $e^{\lambda t}|x(t)|^{2}$, we obtain

$$
\begin{aligned}
e^{\lambda t}|x(t)|^{2} & =|\psi(0)|^{2}+\lambda \int_{0}^{t} e^{\lambda s}\left|x_{s}\right|^{2} d s+2 \int_{0}^{t} e^{\lambda s}\left\langle A x_{s}, x_{s}\right\rangle d s \\
& \left.+\int_{0}^{t} e^{\lambda s} \operatorname{tr}(B(x(s)-x(\rho(s)))) W B(x(s)-x(\rho(s)))\right)^{*} d s \\
& +2 \int_{0}^{t} e^{\lambda s}\left(x_{s}, B(x(s)-x(\rho(s))) d w_{s}\right) \\
& \leq|\psi(0)|^{2}+\lambda \int_{0}^{t} e^{\lambda s}\left|x_{s}\right|^{2} d s+2 \int_{0}^{t} e^{\lambda s}\left\langle A x_{s}, x_{s}\right\rangle d s \\
& +\operatorname{tr}(W) \int_{0}^{t} e^{\lambda s}|B(x(s)-x(\rho(s)))|^{2} d s \\
& +2 \int_{0}^{t} e^{\lambda s}\left(x(s), B(x(s)-x(\rho(s))) d w_{s}\right) .
\end{aligned}
$$

The hypothesis (coerc), (3.1) and the preceding change of variables imply

$$
\begin{aligned}
e^{\lambda t} E|x(t)|^{2}= & E|\psi(0)|^{2}+\lambda \int_{0}^{t} e^{\lambda s} E\left|x_{s}\right|^{2} d s+2 \int_{0}^{t} e^{\lambda s} E\left\langle A x_{s}, x_{s}\right\rangle d s \\
& +\operatorname{tr}(W) \int_{0}^{t} e^{\lambda s} E|B(x(s)-x(\rho(s)))|^{2} d s \\
\leq & \|\psi\|_{1}^{2}+(\lambda+|\nu|) \int_{0}^{t} e^{\lambda s} E\left|x_{s}\right|^{2} d s \\
& +b^{2} \operatorname{tr}(W) \int_{0}^{t} e^{\lambda s} E|x(s)-x(\rho(s))|^{2} d s \\
\leq & \|\psi\|_{1}^{2}+\left(\lambda+|\nu|+2 b^{2} \operatorname{tr}(W)\right) \int_{0}^{t} e^{\lambda s} E|x(s)|^{2} d s \\
& +2 b^{2} \operatorname{tr}(W) \int_{0}^{t} e^{\lambda s} E|x(\rho(s))|^{2} d s \\
\leq & k_{2}\|\psi\|_{1}^{2}+k_{3} \int_{0}^{t} e^{\lambda s} E|x(s)|^{2} d s \\
\leq & k_{4}\|\psi\|_{1}^{2}, \forall t \geq 0
\end{aligned}
$$

where $k_{2}, k_{3}, k_{4}$ are positive constants. Clearly, (1.7) follows from (3.8).

\section{FINAL REMARKS}

We give some remarks on our results.

Remark 2. We have proved Theorem 1 when (coerc) holds. However, if (coerc) does not hold but $A$ is the infinitesimal generator of a $C_{0}$-semigroup $\{S(t)\}$ in $H$, we can prove 
that there exists a unique mild solution of $(P C)$ in $C\left(-h, T ; L^{2}(\Omega ; H)\right.$ ) for all $T>0$ (see Ichikawa [8] for a similar situation without delay).

Remark 3. Now, since we can not apply Itô's formula for the mild solution, we have to consider approximating systems (see Ichikawa [8]) having strong solutions. Using these systems, we can deduce that (1.7) also holds for the mild solution of $(P C)$.

Remark 4. When $A$ generates a $C_{0}$-semigroup satisfying $(A 1), B$ is linear, $\rho(t)=t-h$ (i.e. the delay is constant) and there exists the mild solution of $(P C)$, Mao [9] proves that

$$
\limsup _{t \rightarrow \infty} \frac{1}{t} \log E|x(t)|^{2} \leq-\gamma+\frac{3 M|B|^{2} \operatorname{tr}(W)|S(h)-I|^{2} e^{\gamma h}}{1-3 M|B|^{2} \operatorname{tr}(W) h e^{\gamma h}} .
$$

Obviously, when $\lim _{h \rightarrow 0}|S(h)-I|^{2}=0$, that is, when $S(t)$ is uniformly continuous (which is equivalent to the hypothesis that $A$ is a linear bounded operator), he can ensure that for $h$ sufficiently small

$$
\frac{3 M|B|^{2} \operatorname{tr}(W)|S(h)-I|^{2} e^{\gamma h}}{1-3 M|B|^{2} \operatorname{tr}(W) h e^{\gamma h}}<\gamma
$$

and, consequently, he obtains exponential stability. However, when $S(t)$ is a $C_{0}$-semigroup the last limit does not vanish and it might not be possible to choose $h$ so small that (4.1) holds. But, since $|S(t)-I|^{2} \leq 2\left(M e^{-\gamma t}+1\right)$, if we suppose that $B$ is so small that

$$
6 M(M+1)|B|^{2} \operatorname{tr}(W)<\gamma
$$

then, there exists small $h$ such that it follows (4.1). However, Theorem 2 permits us to obtain exponential stability if $4 M|B|^{2} \operatorname{tr}(W)<\gamma$ (observe that $4 M<6 M(M+1)$ ) and $\rho$ is any delay function satisfying (1.2)-(1.3), in particular, for $\rho(t)=t-h$ with any positive $h>0$. Moreover, our result is valid when $B$ is not linear.

Remark 5. In Caraballo [2], a similar technique to that used in Section 3 gives exponential stability of solutions when the perturbation is linear and takes the form $B(x(\rho(t))) d w_{t}$. In fact, assuming (coerc), (1.2)-(1.4), (A1) and (A3), where

$$
M|B|^{2} \operatorname{tr}(W)<\gamma
$$

then, it follows (1.7). 
Now, it is easy to show that this result remains valid when $B$ is non-linear and satisfies (1.1) and we replace $|B|^{2}$ by $b^{2}$ in (A3). Therefore, a result more general than Haussmann's one is obtained. Observe that $(A 3)$ is similar to the hypothesis $\left(H_{2}\right)$ in Haussmann [7]. However, our result also is valid for non-linear and retarded equations.

Remark 6. Following Haussmann [7] (or Ichikawa [8]), we can obtain that (1.7) implies pathwise stability for the solution of $(P C)$. In fact, if we suppose the hypotheses in Theorem 2, one can prove that there exist positive constants $\alpha, \beta$ and a subset $\Lambda \subset \Omega$, with $P(\Lambda)=0$ such that, if $\omega \notin \Lambda$ then, there exists $T(\omega)>0$ such that

$$
|x(t)|^{2} \leq \alpha\|\psi\|_{1}^{2} e^{-\beta t}, \quad \forall t \geq T(\omega) .
$$

Remark \%. We can prove a result which is more general than Theorem 2. We consider the problem

$$
(P C)^{\prime \prime} \quad \begin{cases}x(t) \in I^{2}(-h, T ; H) \cap L^{2}(\Omega ; C(-h, T ; H)), & \forall T>0, \\ d x(t)=A x(t) d t+F(x(t), x(\rho(t))) d w_{t}, & \forall t \in[0, T], \\ x(t)=\psi(t), & \forall t \in[-h, 0],\end{cases}
$$

where $F: H \times H \mapsto \mathcal{L}(K, H)$ satisfies $F(0,0)=0$ and

$$
|F(x, y)-F(\bar{x}, \bar{y})| \leq b(|x-\bar{x}|+|y-\bar{y}|) .
$$

By an easy modification, we can adapt the proofs of Theorem 1 to get that there exists a unique solution of $(P C)^{\prime \prime}$. Now, it follows

TheOREM 3.-Assume (coerc), (1.1) $)^{\prime},(1.2)-(1.4),($ A1) and

$$
4 M b^{2} \operatorname{tr}(W)<\gamma
$$

Then, there exist positive constants $\lambda, C$ such that

$$
E|x(t)|^{2} \leq C\|\psi\|_{1}^{2} e^{-\lambda t}, \quad \forall t \geq 0,
$$

where $x(t)$ is the solution of $(P C)^{\prime \prime}$.

Proof: Replace $B(x(s)-x(\rho(s)))$ by $F(x(s), x(\rho(s)))$ in the proof of Theorem 2 and estimate the integrals in (3.5) and (3.7) using (1.1)'. 
Now, if we let $F(x, y)=B(x-y)$ where $B: H \mapsto \mathcal{L}(K, H)$ satisfies (1.1), then $(P C)^{\prime \prime}$ reduces to $(P C)^{\prime}$ and Theorem 3 gives Theorem 2.

We observe that the equation in $(P C)^{\prime \prime}$ does not depend on the difference $x(t)-x(\rho(t))$ like $(P C)^{\prime}$. In other words, our proof of Theorem 2 does not make use the advantage of the fact that the perturbation is linearly bounded by the difference $x(t)-x(\rho(t))$. Hence it is not surprised to see that Theorem 2 can not apply to the case of bounded $A$ and $\rho(t)=t-h, 4 M b^{2} \operatorname{tr}(W) \geq \gamma$. However, in this case Mao [9] can ensure that for sufficiently small $h,(P C)^{\prime}$ is exponentially stable in mean square. Consequently, we have the following unsolved question:

Is the zero solution of $(P C)^{\prime}$ exponentially stable in mean square if we assume $A$ unbounded, $4 M b^{2} \operatorname{tr}(W) \geq \gamma$ and $t-\rho(t) \leq \rho(0)$ small enough?

\section{EXAMPLE}

In order to illustrate our results, we can consider the following example:

Let $\mathcal{O}$ be a bounded open subset in $\mathbf{R}^{N}$ and let $w_{t}$ be a standard Wiener process (i.e. $K=\mathbf{R}, W=1$ ) over a complete probability space. We consider the Sobolev spaces (see Brezis [1]) $V=H_{0}^{1}(\mathcal{O} ; \mathbf{R}), H=L^{2}(\mathcal{O} ; \mathbf{R})$. We denote by $|\cdot|$ the usual norm in $H$. We also know that the seminorm $\|u\|^{2}=\int_{\mathcal{O}} \sum_{i=1}^{N}\left[\frac{\partial u}{\partial x_{i}}\right]^{2} d x$ is, in fact, a norm in $V$, equivalent to the usual one.

Let $A$ be defined by

$$
A u=\sum_{i=1}^{N} \frac{\partial^{2} u}{\partial x_{i}^{2}}-u=(\Delta-I)(u), \quad \forall u \in V .
$$

Obviously, $A \in \mathcal{L}\left(V, V^{\prime}\right)$ and

$$
\begin{aligned}
-2\langle A u, u\rangle & =-2 \int_{\mathcal{O}} \sum_{i=1}^{N} \frac{\partial^{2} u(x)}{\partial x_{i}^{2}} u(x) d x+2 \int_{\mathcal{O}} u(x)^{2} d x \\
& =2 \int_{\mathcal{O}} \sum_{i=1}^{N}\left[\frac{\partial u(x)}{\partial x_{i}}\right]^{2} d x+2|u|^{2} \\
& =2\|u\|^{2}+2|u|^{2}, \quad \forall u \in V .
\end{aligned}
$$

Therefore, ( $\operatorname{coerc}$ ) holds with $\nu=-2, \varepsilon=2$. Moreover, from Dautray and Lions [6] it 
follows that $A$ is the infinitesimal generator of a $C_{0}$-semigroup $S(t)$ in $H$ such that

$$
|S(t)|^{2} \leq e^{-2 t}
$$

and $(A 1)$ is satisfied taking $M=1, \gamma=2$.

Finally, we consider $B: H \rightarrow H$ defined by

$$
B(u)=\frac{b u}{1+|u|}, \quad \text { where } b \in L^{\infty}(\mathcal{O} ; \mathbf{R}) .
$$

It is easy to see that

$$
|B(u)-B(v)| \leq\|b\|_{L^{\infty}(\mathcal{O})}|u-v|
$$

and so, it follows (1.1) with $b=\|b\|_{L^{\infty}(\mathcal{O})}$.

Consequently, for each $\psi \in I^{2}(-h, 0 ; V) \cap L^{2}(\Omega ; C(-h, 0 ; H))$, and each $\rho$ satisfying (1.2)-(1.3), there exists a unique solution of $(P C)$. If, in addition, $b=\|b\|_{L^{\infty}(\mathcal{O})}<1 / 2$, then (1.7) holds (observe that (4.2) assures exponential stability provided $b=\|b\|_{L^{\infty}(\mathcal{O})}<1 / 6$ and $h$ small enough).

\section{ACKNOWLEDGEMENTS}

We wish to express our thanks to the referees for their useful and detailed suggestions.

\section{REFERENCES}

[1] H. Brezis, Analyse Fonctionnelle, Masson, Paris, 1983.

[2] T. Caraballo, 'Asymptotic Exponential Stability of Stochastic Partial Differential Equations with Delay', Stochastics and Stochastics Reports $\underline{33}$ (1990), 27-47.

[3] A. Chojnowska-Michalik, Stochastic Differential Equations in Hilbert Spaces and Their Applications, Thesis, Institute of Mathematics, Polish Academy of Sciences, 1976.

[4] P. L. Chow, 'Stability of nonlinear stochastic evolution equations', J. Math. Anal. Appl. $\underline{89}$ (1982), 400-419.

[5] R. F. Curtain and A. J. Pritchard, Infinite dimensional linear systems theory, Lecture Notes in Control and Information Sciences $\underline{8}$, Springer-Verlag, Berlin, 1978.

[6] R. Dautray and J. L. Lions, Analyse Mathèmatique et Calcul Numérique pour les Sciences et les Techniques, Masson, Paris, 1984.

[7] U. G. Haussmann, 'Asymptotic Stability of the Linear Ito Equation in Infinite Dimensions', J. Math. Anal. Appl. $\underline{65}$ (1978), 219-235. 
[8] A. Ichikawa, 'Stability of Semilinear Stochastic Evolution Equations', J. Math. Anal. Appl. $\underline{90}$ (1982), 12-44.

[9] X. Mao, 'Exponential stability for stochastic differential delay equations in Hilbert spaces', Quart. J. Math. (2) $\underline{42}$ (1991), 77-85.

[10] X. Mao, 'Exponential stability in mean square for stochastic differential equations', Stoch. Anal. Appl. $\underline{8}$ (1) (1990), 91-103.

[11] E. Pardoux, Intégrales Stochastiques Hilbertiennes, Rapport de Recherche CEREMADE (University of Paris-Dauphine) vol. $\underline{7617}$ (1976).

[12] E. Pardoux, 'Stochastic Partial Differential Equations and Filtering of Diffusion Processes', Stochastics $\underline{3}$ (1979),127-167.

[13] J. Real, 'Stochastic Partial Differential Equations with Delays', Stochastics $\underline{8}$ (1982), $81-102$.

[14] J. Zabczyk, On stability of infinite dimensional stochastic systems, Probability Theory, Z. Ciesislki (Ed.), Banach Center Publications, vol. 모, Warsaw, 1979, 273-281. 